\title{
BURGERS' TURBULENCE PROBLEM WITH LINEAR OR QUADRATIC EXTERNAL POTENTIAL
}

\author{
O. E. BARNDORFF-NIELSEN, ${ }^{*}$ University of Aarhus \\ N. N. LEONENKO, ${ }^{* *}$ Cardiff University
}

\begin{abstract}
We consider solutions of Burgers' equation with linear or quadratic external potential and stationary random initial conditions of Ornstein-Uhlenbeck type. We study a class of limit laws that correspond to a scale renormalization of the solutions.

Keywords: Inhomogeneous Burgers equation; Ornstein-Uhlenbeck-type process; linear potential; quadratic potential; random initial condition; stationary process; short-range dependence; long-range dependence; infinitely divisible distribution; normal inverse Gaussian distribution
\end{abstract}

2000 Mathematics Subject Classification: Primary 62M40; 62M15; 60H15; $60 \mathrm{G60}$

\section{Introduction}

We consider the one-dimensional Burgers equation of the form

$$
u_{t}+u u_{x}=\mu u_{x x}+2 \mu \Phi_{x}
$$

subject to the initial condition

$$
u(0, x)=u_{0}(x),
$$

where $u \equiv u(t, x), t>0, x \in \mathbb{R}$, is a velocity field; $\Phi \equiv \Phi(x), x \in \mathbb{R}$, is the external potential; $\Phi_{x} \equiv(\mathrm{d} / \mathrm{d} x) \Phi(x), \mu>0$, is the viscosity of the media (the reciprocal of which, $\operatorname{Re}=1 / \mu$, corresponds to the Reynolds number); and we define

$$
U \equiv U(x)=\int^{x} u_{0}(\xi) \mathrm{d} \xi
$$

to be the velocity potential. Note that subscripts on a function of a single variable denote total differentiation, while those on a function of many variables denote the appropriate partial derivative. The nonlinear equation (1) can be viewed as a simplified version of the NavierStokes equation. However, the differences between the Burgers and Navier-Stokes equations are as interesting as the similarities.

Our interest is in cases where the external potential $\Phi$ is a linear or quadratic function and the initial condition

$$
u_{0}(x)=\xi(x), \quad x \in \mathbb{R},
$$

Received 12 December 2003; revision received 6 December 2004.

* Postal address: Department of Mathematical Sciences, University of Aarhus, Ny Munkegade, K-8000 Aarhus C, Denmark. Email address: oebn@imf.au.dk

** Postal address: Cardiff School of Mathematics, Cardiff University, Senghennydd Road, Cardiff CF24 4AG, UK.

Email address: leonenkon@ cardiff.ac.uk 
is a stationary random process with short- or long-range dependence, and which has sample paths that are integrable over all finite intervals. The initial-value problem (1), (4) is known as Burgers' turbulence problem.

Our main interest is in the analysis of the limiting behavior, as $\varepsilon \rightarrow 0$, of the average solutions

$$
\frac{1}{A_{\varepsilon}} \int_{0}^{x / \varepsilon}[u(t, y)-B(t, y)] \mathrm{d} y, \quad x>0,
$$

with $t>0$ and $x \in \mathbb{R}$ fixed, and where $A_{\varepsilon}$ is a normalizing factor and $B(t, y)$ is a centering function.

The Burgers equation constitutes an important example of the nonlinear partial differential equations studied in turbulence (see, for example, Burgers (1974), Chorin (1975), Kardar et al. (1986), Shandarin and Zeldovich (1989), Gurbatov et al. (1991), and Frisch (1995)) and finance (see Hodges and Carverhill (1993) and Hodges and Selby (1997)).

Burgers' equation with random initial conditions and external potential $\Phi=0$ has been extensively studied in the last fifteen years by Rosenblatt (1987), Bulinskil and Molchanov (1991), Funaki et al. (1995), Leonenko and Orsingher (1995), Molchanov et al. (1995), Hu and Woyczyński (1995), Bertoin (1998a), (1998b), Leonenko and Woyczyński (1998), Ryan (1998), and Dermone et al. (1999), among others. Recent books of Woyczyński (1998) and Leonenko (1999) contain a complete bibliography of the subject and expositions of some of the principal results of the theory of Burgers' turbulence.

In particular, Bulinskiǐ and Molchanov (1991), Albeverio et al. (1994), Leonenko and Li (1994), Leonenko and Orsingher (1995), Funaki et al. (1995), and Deriev and Leonenko (1997) obtained Gaussian and non-Gaussian scenarios for parabolically rescaled solutions of the Burgers equation with weakly dependent and strongly dependent random initial conditions. These scenarios are in some sense subordinate to the Gaussian white noise random measure.

For other approaches to Burgers' turbulence problem, see Rosenblatt (1987), Hu and Woyczyński (1995) (for asymptotic distributions of averages of solutions of Burgers equation with random data), Sinai (1992) (for statistics of shocks and related topics), Molchanov et al. (1995) (for hyperbolic asymptotics), Bertoin (1998a), (1998b) and Ryan (1998) (for the large deviation principle and statistics of shock waves), and their references.

In a sense, the Gaussian and non-Gaussian scenarios for a solution of Burgers' equation with random initial condition are results of the application of the central limit theorem or noncentral limit theorems for nonlinear functionals of Gaussian processes and fields. The results of Taqqu (1975), (1979) and Dobrushin and Major (1979) contain a main principle for the study of limiting distributions of nonlinear transformations of Gaussian processes with long-range dependence. These distributions are not always Gaussian, but they are subordinate to Gaussian white noise random measures. For a recent development, see Anh and Leonenko (1999) and its references.

Recent work of Barndorff-Nielsen (1998a), (1998b), (2001) and Barndorff-Nielsen and Pérez-Abreu (1999) presented a new type of process with long-range dependence and possibly non-Gaussian marginal distributions such as gamma, inverse Gaussian, or normal inverse Gaussian distributions (which are all infinitely divisible). These processes are constructed via stochastic integrals with respect to independently scattered random measures with infinitely divisible distributions.

Our paper is motivated by the papers of Rosenblatt (1987), Hu and Woyczyński (1995) and Barndorff-Nielsen (1998a), (1998b), (2001). We present an explicit solution to Burgers' turbulence problem with linear or quadratic external potential. Furthermore, we study the limiting behavior of averages of solutions of Burgers' equation with linear or quadratic 
external potential and Ornstein-Uhlenbeck-type initial conditions with short- and long-range dependence.

Note that a more hydrodynamic and physical approach to the forced version of Burgers' turbulence problem was proposed in a paper by Saichev and Woyczyński (1997), in which statistical properties of Burgers' turbulence problem with random potential were studied.

Throughout the paper, as standard notation we shall write $\mathrm{C}\{\zeta \neq y\}$ for the cumulant function of a random variable $y$, i.e.

$$
\mathrm{C}\{\zeta \neq y\}=\log \mathrm{E} \exp \{\mathrm{i} \zeta y\} .
$$

\section{Cole-Hopf solutions of the Burgers equation}

The Burgers equation (1) can be reduced to a parabolic-type equation using the Hopf-Cole transformation

$$
u(t, x)=-2 \mu \frac{\partial}{\partial x} \log h(t, x)
$$

(see, e.g. Witham (1974), Rosenblatt (1987), Gurbatov et al. (1991), Albeverio et al. (1994), or Bertini and Cancrini (1995)), which reduces (1) and (2) to the equation

$$
h_{t}=\mu h_{x x}-\Phi h
$$

subject to the initial condition

$$
h(0, x)=h_{0}(x)=\exp \left\{-\frac{U(x)}{2 \mu}\right\}=\exp \left\{-\frac{1}{2 \mu} \int^{x} u_{0}(\xi) \mathrm{d} \xi\right\},
$$

where $h \equiv h(t, x), t>0, x \in \mathbb{R}$.

The parabolic-type equation (6) reduces, for $\Phi=0$, to the classical heat equation

$$
h_{t}=\mu h_{x x},
$$

which has a fundamental solution of the following form:

$$
g(t, x)=\frac{1}{\sqrt{4 \pi \mu t}} \exp \left\{-\frac{x^{2}}{4 \mu t}\right\}, \quad t>0, x \in \mathbb{R} .
$$

Thus, the field

$$
u(t, x)=\frac{\int_{\mathbb{R}}((x-y) / t) g(t, x-y) \mathrm{e}^{-U(y) / 2 \mu} \mathrm{d} y}{\int_{\mathbb{R}} g(t, x-y) \mathrm{e}^{-U(y) / 2 \mu} \mathrm{d} y}=: \frac{I(t, x)}{J(t, x)}
$$

solves the initial-value problem (1)-(3) in the case where the external potential vanishes.

We recall Hopf's formulation of this result (see Hopf (1950)).

Theorem 1. Let $u(t, x)$ be a solution of (1) with locally integrable initial condition $u(0, x)=$ $u_{0}(x)$ and external potential $\Phi=0$. If

$$
\int_{0}^{x} u_{0}(\xi) \mathrm{d} \xi=o\left(x^{2}\right)
$$

as $|x| \rightarrow \infty$, then

$$
u(t, x)=\frac{\int_{\mathbb{R}}((x-y) / t) \exp \{-(1 / 2 \mu) F(x, y, t)\} \mathrm{d} y}{\int_{\mathbb{R}} \exp \{-(1 / 2 \mu) F(x, y, t)\} \mathrm{d} y},
$$


where

$$
F(x, y, t)=\frac{(x-y)^{2}}{2 t}+\int_{0}^{y} u_{0}(\xi) \mathrm{d} \xi=\frac{(x-y)^{2}}{2 t}+U(y)
$$

is a solution of (1), with $\Phi=0$, that satisfies the initial condition

$$
\int_{0}^{x} u(y, t) \mathrm{d} y \rightarrow \int_{0}^{a} u_{0}(y) \mathrm{d} y \quad \text { as } x \rightarrow a, t \rightarrow 0,
$$

for every a. If, in addition, $u_{0}(x)$ is continuous at $x=a$, then

$$
u(x, t) \rightarrow u_{0}(a) \quad \text { as } x \rightarrow a, t \rightarrow 0 .
$$

We note that (6), with initial condition

$$
h(0, x)=h_{0}(x)
$$

can be solved explicitly in a number of cases (see, e.g. Kochmanski (1994) or Leonenko and Mel'nikova (2001)). In particular, for the linear external potential

$$
\Phi(x)=a+b x
$$

the field

$$
h(t, x)=\exp \left\{-t(a+b x)+\frac{\mu b^{2} t^{3}}{3}\right\} \int_{\mathbb{R}} \frac{\exp \left\{-\left(x-y-b \mu t^{2}\right)^{2} / 4 \mu t\right\}}{\sqrt{4 \pi \mu t}} h_{0}(y) \mathrm{d} y
$$

solves the initial-value problem (6), (8), and, under the conditions of Theorem 1, the field

$$
u(t, x)=2 \mu b t+\frac{\int_{\mathbb{R}}\left(\left(x-y-b \mu t^{2}\right) / t\right) \exp \left\{-\left(x-y-b \mu t^{2}\right)^{2} / 4 \mu t\right\} \mathrm{e}^{-U(y) / 2 \mu} \mathrm{d} y}{\int_{\mathbb{R}} \exp \left\{-\left(x-y-b \mu t^{2}\right)^{2} / 4 \mu t\right\} \mathrm{e}^{-U(y) / 2 \mu} \mathrm{d} y}
$$

solves the initial-value problem (1)-(3).

For the quadratic external potential

$$
\Phi(x)=a+b x^{2}, \quad b>0,
$$

the field

$$
\begin{aligned}
h(t, x)= & \exp \left\{-a t-\frac{\sqrt{b} x^{2}}{\sqrt{4 \mu}} \tanh (\omega t)\right\} \\
& \times \int_{\mathbb{R}} \frac{\exp \left\{-[x-y \cosh (\omega t)]^{2} / \sqrt{\mu / b} \sinh (2 \omega t)\right\} h_{0}(y) \mathrm{d} y}{[2 \pi \sqrt{\mu / b} \sinh (\omega t)]^{1 / 2}}
\end{aligned}
$$

solves the initial-value problem (6),(8), where

$$
\omega=2 \sqrt{\mu b}
$$

Note that for $a=0$ and $b=1,(12)$ can be reduced to Mehler's formula. (For information on Mehler's formula see, e.g. Cycon et al. (1987, pp. 286-287) or Berline et al. (1992, p. 154)). 
Hence, under the conditions of Theorem 1, the solution to (1)-(3) with external potential (11) can be written as

$$
\begin{aligned}
u(t, x)=2 & \sqrt{\mu b} x \tanh (\omega t) \\
+ & 4 \mu\left(\int_{\mathbb{R}} \frac{x-y \cosh (\omega t)}{\sqrt{\mu / b} \sinh (2 \omega t)} \exp \left\{-\frac{[x-y \cosh (\omega t)]^{2}}{\sqrt{\mu / b} \sinh (2 \omega t)}-\frac{U(y)}{2 \mu}\right\} \mathrm{d} y\right) \\
& \times\left(\int_{\mathbb{R}} \exp \left\{-\frac{[x-y \cosh (\omega t)]^{2}}{\sqrt{\mu / b} \sinh (2 \omega t)}-U(y) / 2 \mu\right\} \mathrm{d} y\right)^{-1} .
\end{aligned}
$$

Remark 1. For an external potential $\Phi$ of general (possibly random) form, the solution of Burgers' initial-value problem can be expressed in terms of the Feynman-Kac formula (see, e.g. Holden et al. (1996), Molchanov et al. (1997), Woyczyński (1998), and Leonenko (1999), among others), or in terms of functional integrals (see Saichev and Woyczyński (1997, Equation (12))).

\section{Rosenblatt's type-reduction principles}

Now, consider the Burgers equation (1) with external potential $\Phi=0$ and random initial condition

$$
u_{0}(x)=\xi(x), \quad x \in \mathbb{R} .
$$

We introduce the following two conditions.

Condition A. The function $\xi(x), x \in \mathbb{R}$, is a strictly stationary random process whose sample paths are almost surely locally integrable and such that $\mathrm{E}|\xi(x)|<\infty$.

Under this condition, we have

$$
\int_{0}^{x} \xi(y) d y=O(x)
$$

as $|x| \rightarrow \infty$ and, consequently, condition (7) in Hopf's theorem is satisfied.

Condition $\mathbf{A}^{\prime} . \mathrm{E} \xi^{2}(x)<\infty$ and

$$
A_{\varepsilon}^{2}=\operatorname{var}\left[\int_{0}^{1 / \varepsilon} \xi(y) \mathrm{d} y\right] \rightarrow \infty
$$

as $\varepsilon \rightarrow 0$.

Rosenblatt (1987) observed that, for each $\varepsilon>0$,

$$
\frac{1}{2 \mu} \int_{0}^{1 / \varepsilon} u(t, x) \mathrm{d} x=\frac{1}{2 \mu} \int_{0}^{1 / \varepsilon} \xi(x) \mathrm{d} x-R_{\varepsilon},
$$

where $t>0$ is fixed and

$$
R_{\varepsilon}=\log \left\{\int_{\mathbb{R}} \exp \left\{-v^{2} / 4 \mu t+\varsigma_{\varepsilon}(v)\right\} \mathrm{d} v\right\}-\log \left\{\int_{\mathbb{R}} \exp \left\{-v^{2} / 4 \mu t+\varsigma(v)\right\} \mathrm{d} \nu\right\},
$$

with

$$
\varsigma_{\varepsilon}(v)=-\frac{1}{2 \mu} \int_{1 / \varepsilon}^{(1 / \varepsilon)+v} \xi(y) \mathrm{d} y, \quad \varsigma(v)=-\frac{1}{2 \mu} \int_{0}^{v} \xi(y) \mathrm{d} y .
$$


The random variables $\zeta_{\varepsilon}(v)$ and $\zeta(v)$ are identically distributed. Thus, under Conditions A and $\mathrm{A}^{\prime}$,

$$
\frac{1}{A_{\varepsilon}}\left(\int_{0}^{x / \varepsilon} u(t, y) \mathrm{d} y-\int_{0}^{x / \varepsilon} \xi(y) \mathrm{d} y\right) \rightarrow 0
$$

in probability as $\varepsilon \rightarrow 0$, where $u(t, y), t>0, y \in \mathbb{R}$, is the solution of Burgers' equation with initial condition (14). This leads to the so-called Rosenblatt reduction principle (see Rosenblatt (1987)).

Theorem 2. Under Conditions $A$ and $A^{\prime}$, the limiting behavior (as $\varepsilon \rightarrow 0$ ) of the average process

$$
\frac{1}{A_{\varepsilon}} \int_{0}^{x / \varepsilon}[u(t, y)-\mathrm{E} \xi(y)] \mathrm{d} y, \quad x>0,
$$

is the same as that of the average process

$$
\frac{1}{A_{\varepsilon}} \int_{0}^{x / \varepsilon}[\xi(y)-\mathrm{E} \xi(y)] \mathrm{d} y, \quad x>0,
$$

in the sense of finite-dimensional distributions.

The limiting behavior of the process (17) itself has been studied in many cases.

Remark 2. Theorem 2 implies that the low-frequency behavior of the spectrum of $u(t, x)$ in the immediate neighborhood of 0 is independent of $t>0$ and is the same as that of $\xi(x)$. Similar remarks, also suggesting this, have been made in the case of homogeneous turbulence (see Batchelor (1953, Section 3.3)).

Now consider the Burgers equation (1) with linear external potential $\Phi$ of the form (9) and initial condition (14). Observe that in this case, from (5), we have

$$
\frac{1}{2 \mu} \int_{0}^{1 / \varepsilon} u(t, x) \mathrm{d} x=-\log \frac{h(t, 1 / \varepsilon)}{h(t, 0)},
$$

where $h(t, u)$ is defined as in (10) and

$$
\begin{aligned}
h\left(t, \frac{1}{\varepsilon}\right)= & \exp \left\{-t\left(a+\frac{b}{\varepsilon}\right)+\frac{\mu b^{2} t^{3}}{3}\right\} \int_{\mathbb{R}} h_{0}\left(v+\frac{1}{\varepsilon}\right) \frac{\exp \left\{-\left(v-b \mu t^{2}\right)^{2} / 4 \mu t\right\}}{\sqrt{4 \pi t \mu}} \mathrm{d} v, \\
h_{0}\left(v+\frac{1}{\varepsilon}\right)= & \exp \left\{-\frac{1}{2 \mu} \int_{0}^{v+1 / \varepsilon} \xi(x) \mathrm{d} x\right\} \\
= & \exp \left\{-\frac{1}{2 \mu} \int_{0}^{1 / \varepsilon} \xi(x) \mathrm{d} x\right\} \exp \left\{-\frac{1}{2 \mu} \int_{1 / \varepsilon}^{v+1 / \varepsilon} \xi(x) \mathrm{d} x\right\} \\
h(t, 0)= & \exp \left\{-t a+\frac{\mu b^{2} t^{3}}{3}\right\} \\
& \times \int_{\mathbb{R}} \exp \left\{-\frac{1}{2 \mu} \int_{0}^{v} \xi(x) \mathrm{d} x\right\} \exp \left\{-\frac{\left(v-b \mu t^{2}\right)^{2}}{4 \mu t}\right\} \frac{\mathrm{d} v}{\sqrt{4 \pi \mu t}} .
\end{aligned}
$$

Thus, for every $\varepsilon>0$ and $t>0$,

$$
\frac{1}{2 \mu} \int_{0}^{1 / \varepsilon} u(t, x) \mathrm{d} x-\frac{t b}{\varepsilon}=\frac{1}{2 \mu} \int_{0}^{1 / \varepsilon} \xi(x) \mathrm{d} x-R_{\varepsilon}^{\prime},
$$


where

$$
\begin{aligned}
R_{\varepsilon}^{\prime}= & \log \left\{\int_{\mathbb{R}} \exp \left\{-\frac{\left(\nu-b \mu t^{2}\right)^{2}}{4 \mu t}+\varsigma_{\varepsilon}(\nu)\right\} \mathrm{d} \nu\right\} \\
& -\log \left\{\int_{\mathbb{R}} \exp \left\{-\frac{\left(\nu-b \mu t^{2}\right)^{2}}{4 \mu t}+\varsigma(\nu)\right\} \mathrm{d} \nu\right\}
\end{aligned}
$$

and the identically distributed random variables $\varsigma_{\varepsilon}(v)$ and $\varsigma(v)$ are defined as in (15). We arrive at the following generalization of Rosenblatt's reduction principle in the presence of a linear external potential $\Phi$ of the form (9).

Theorem 3. Let $u(t, x), t>0, x \in \mathbb{R}$, be a solution of the initial-value problem in (1) and (14) with linear external potential $\Phi$ of the form (9). Suppose that Conditions A and $A^{\prime}$ are satisfied. Then, the limiting behavior (as $\varepsilon \rightarrow 0$ ) of the average process

$$
\frac{1}{A_{\varepsilon}} \int_{0}^{x / \varepsilon}[u(t, y)-2 t b \mu-\mathrm{E} \xi(y)] \mathrm{d} y, \quad x>0,
$$

is the same as that of the average process

$$
\frac{1}{A_{\varepsilon}} \int_{0}^{x / \varepsilon}[\xi(y)-\mathrm{E} \xi(y)] \mathrm{d} y
$$

in the sense of finite-dimensional distributions.

Next, consider the Burgers equation (1) with quadratic external potential $\Phi$ of the form (11). In this case, using (5) again, we obtain the following identity:

$$
\frac{1}{2 \mu} \int_{0}^{1 / \varepsilon} u(t, x) \mathrm{d} x=-\log \frac{h(t, 1 / \varepsilon)}{h(t, 0)},
$$

where now $h(t, u)$ is defined as in (11) and, consequently,

$$
\begin{aligned}
h\left(t, \frac{1}{\varepsilon}\right)= & \exp \left\{-a t-\sqrt{b}\left(\frac{1}{\varepsilon}\right)^{2} \frac{\tanh (\omega t)}{\sqrt{4 \mu}}\right\} \\
& \times \int_{\mathbb{R}} h_{0}\left(\frac{v+1 / \varepsilon}{\cosh (\omega t)}\right) \exp \left\{-\frac{v^{2}}{\sqrt{\mu / b} \sinh (2 \omega t)}\right\} \mathrm{d} v, \\
h_{0}\left(\frac{v+1 / \varepsilon}{\cosh (\omega t)}\right)= & \exp \left\{-\frac{1}{2 \mu} \int_{0}^{1 / \varepsilon \cosh (\omega t)} \xi(x) \mathrm{d} x\right\} \\
& \times \exp \left\{-\frac{1}{2 \mu} \int_{1 / \varepsilon \cosh (\omega t)}^{(1 / \varepsilon+v) / \cosh (\omega t)} \xi(x) \mathrm{d} x\right\}, \\
h(t, 0)= & \exp \{-a t\} \int_{\mathbb{R}} \exp \left\{-\frac{1}{2 \mu} \int_{0}^{v / \varepsilon \cosh (\omega t)} \xi(x) \mathrm{d} x\right\} \\
& \times \exp \left\{-\frac{v^{2}}{\sqrt{\mu / b} \sinh (2 \omega t)}\right\} \mathrm{d} v .
\end{aligned}
$$

Therefore, for every $\varepsilon>0$ and $t>0$,

$$
\frac{1}{2 \mu} \int_{0}^{1 / \varepsilon} u(t, x) \mathrm{d} x=\sqrt{\frac{b}{4 \mu}} \tanh (\omega t) \frac{1}{\varepsilon^{2}}+\frac{1}{2 \mu} \int_{0}^{1 / \varepsilon \cosh (\omega t)} \xi(x) \mathrm{d} x-R_{\varepsilon}^{\prime \prime},
$$


where

$$
\begin{aligned}
R_{\varepsilon}^{\prime \prime}= & \log \left\{\int_{\mathbb{R}} \exp \left\{-\frac{v^{2}}{\sqrt{\mu / b} \sinh (2 \omega t)}+\tilde{\zeta}_{\varepsilon}(v)\right\} \mathrm{d} \nu\right\} \\
& -\log \left\{\int_{\mathbb{R}} \exp \left\{-\frac{v^{2}}{\sqrt{\mu / b} \sinh (2 \omega t)}+\tilde{\zeta}(\nu)\right\} \mathrm{d} \nu\right\},
\end{aligned}
$$

and the random variables $\tilde{\zeta}_{\varepsilon}(v)$ and $\tilde{\zeta}(v)$ are identically distributed and defined as

$$
\tilde{\zeta}_{\varepsilon}(\nu)=-\frac{1}{2 \mu} \int_{1 / \varepsilon \cosh (\omega t)}^{(1+\varepsilon v) / \varepsilon \cosh (\omega t)} \xi(y) \mathrm{d} y, \quad \tilde{\zeta}_{v}=-\frac{1}{2 \mu} \int_{0}^{\nu / \cosh (\omega t)} \xi(y) \mathrm{d} y,
$$

with $\omega$ as defined in (13).

We arrive at the following result.

Theorem 4. Let $u(t, x), t>0, x \in \mathbb{R}$, be a solution of initial-value problem (1), (14), with quadratic external potential $\Phi$ of the form (11). Suppose that Conditions $A$ and $A^{\prime}$ are satisfied. Then, the limiting behavior (as $\varepsilon \rightarrow 0$ ) of the average process

$$
\frac{1}{A_{\varepsilon}} \int_{0}^{x / \varepsilon}\left[u(t, y)-2 y \sqrt{b \mu} \tanh (\omega t)-\frac{\mathrm{E} \xi(y)}{\cosh (\omega t)}\right] \mathrm{d} y, \quad x \geq 0,
$$

is the same as that of the average process

$$
\frac{1}{A_{\varepsilon}} \int_{0}^{[x / \cosh (\omega t)](1 / \varepsilon)}[\xi(y)-\mathrm{E} \xi(y)] \mathrm{d} y, \quad x \geq 0,
$$

in the sense of finite-dimensional distributions.

\section{Self-decomposability and OU processes}

This section mainly reviews a number of results discussed in Barndorff-Nielsen (1998a), (2001).

\subsection{Infinite divisibility}

Recall that a random variable $y$ is infinitely divisible if its cumulant function has the LévyKhinchin representation

$$
\mathrm{C}\{\zeta \ddagger y\}=\mathrm{i} a \zeta-\frac{1}{2} b \zeta^{2}+\int_{\mathbb{R}}\left(\mathrm{e}^{\mathrm{i} \zeta u}-1-\mathrm{i} \zeta \tau(u)\right) Q(\mathrm{~d} u), \quad \zeta \in \mathbb{R},
$$

where $a \in \mathbb{R}, b>0$, and

$$
\tau(u)= \begin{cases}u & \text { if }|u| \leq 1, \\ u /|u| & \text { if }|u|>1,\end{cases}
$$

and where the Lévy measure $Q$ is a Radon measure on $\mathbb{R} \backslash\{0\}$ such that $Q(\{0\})=0$ and

$$
\int_{\mathbb{R}} \min \left(1, u^{2}\right) Q(\mathrm{~d} u)<\infty .
$$

Example 1. (Gaussian distribution.) The random variable $y$ has the normal distribution $\mathrm{N}(a, b)$ if $Q=0$ in the representation (22). 
Example 2. (Normal inverse Gaussian distribution.) The density function of an $\operatorname{NIG}(\alpha, \beta$, $\mu, \delta$ ) random variable $y$ is given by

$$
\operatorname{nig}(u)=\frac{\alpha}{\pi} \exp \left\{\delta \sqrt{\alpha^{2}-\beta^{2}}+\beta(u-\mu)\right\} \frac{K_{1}(\delta \alpha g((u-\mu) / \delta))}{g((u-\mu) / \delta)}, \quad u \in \mathbb{R},
$$

where $\delta>0,0 \leq|\beta|<\alpha, g(x)=\left(1+x^{2}\right)^{1 / 2}$, and $K_{v}$ is the modified Bessel function of the third kind of order $v$ (see, e.g. Watson (1944)). The distribution is symmetric around $\mu$, provided that $\beta=0$. The normal distribution $\mathrm{N}\left(\mu, \sigma^{2}\right)$ appears as a limiting case for $\beta=0$, $\alpha \rightarrow \infty$, and $\delta / \alpha=\sigma^{2}$, and the Cauchy law appears as limiting case of $\operatorname{NIG}(\alpha, 0,0,1)$ for $\alpha \rightarrow 0$. Note that

$$
\mathrm{E} y=\mu+\delta \rho /\left(1-\rho^{2}\right)^{1 / 2}, \quad \operatorname{var} y=\delta^{2} / \bar{\alpha}\left(1-\rho^{2}\right)^{3 / 2},
$$

where $\rho=\beta / \alpha$ and $\bar{\alpha}=\delta \alpha$. If $y_{1}, \ldots, y_{m}$ are independent $\operatorname{NIG}\left(\alpha, \beta, \mu_{i}, \delta_{i}\right), i=1, \ldots, m$, random variables, then

$$
y_{1}+\cdots+y_{m} \sim \operatorname{NIG}\left(\alpha, \beta, \mu_{1}+\cdots+\mu_{m}, \delta_{1}+\cdots+\delta_{m}\right) .
$$

We also note that $\operatorname{NIG}(\alpha, \beta, \delta, \mu)$ has semiheavy tails, specifically,

$$
\operatorname{nig}(u) \sim \text { const } \times|u|^{-3 / 2} \exp \{-\alpha|u|+\beta u\} \quad \text { as } u \rightarrow \pm \infty .
$$

Assuming that $\mu=0$, we obtain the cumulant function of the $\operatorname{NIG}(\alpha, \beta, \mu, \delta)$ distribution in the Lévy-Khinchin form:

$$
\begin{aligned}
\mathrm{C}\{\zeta \ddagger y\}= & \int_{|u| \geq 1}\left(\mathrm{e}^{\mathrm{i} \zeta u}-1\right) f(u ; \alpha, \beta, \delta) \mathrm{d} u \\
& +\int_{|u|<1}\left(\mathrm{e}^{\mathrm{i} \zeta u}-1-\mathrm{i} \zeta u\right) f(u ; \alpha, \beta, \delta) \mathrm{d} u \\
& +\frac{2 \mathrm{i} \zeta}{\pi} \delta \alpha \int_{0}^{1} \sinh (\beta x) K_{1}(\alpha x) \mathrm{d} x
\end{aligned}
$$

where

$$
f(u ; \alpha, \beta, \delta)=\pi^{-1} \delta \alpha|u|^{-1} \mathrm{e}^{\beta u} K_{1}(\alpha|u|) .
$$

A stochastic process $z(s), s \geq 0$, is said to be a Lévy process if it has independent increments, càdlàg sample paths, and is continuous in probability. If the increments are stationary, $z$ is said to be homogeneous. In the following, we take the term 'Lévy process' to mean a homogeneous Lévy process $z$ such that $z(s) \stackrel{\mathrm{P}}{\rightarrow} 0$ as $s \downarrow 0$.

If $z$ is a Lévy process then the cumulant function $z$ satisfies $\mathrm{C}\{\zeta \doteqdot z(s)\}=s \mathrm{C}\{\zeta \doteqdot z(1)\}$. Note that to any infinitely divisible random variable $y$ there corresponds a Lévy process $z$ such that $y \stackrel{L}{=} z(1)$; we refer to $z$ as the Lévy process generated by $y$, where $\stackrel{L}{=}$ means equality in law. If $y$ has representation (22), then $(a, b, Q)$ will be called the characteristics of the Lévy process $z(s), s \geq 0$.

\subsection{Self-decomposability}

An infinitely divisible random variable $y$ (or its law) is self-decomposable if its characteristic function $\phi(t)=\operatorname{E} \exp \{\mathrm{i} t y\}, t \in \mathbb{R}$, has the property that, for every $c \in(0,1)$, there exists a characteristic function $\phi_{c}$ such that $\phi(t)=\phi(c t) \phi_{c}(t)$ for all $t \in \mathbb{R}$. Equivalently, $y$ is selfdecomposable if its Lévy measure is $Q(\mathrm{~d} u)=q(u) \mathrm{d} u$ with $q(u)=|u|^{-1} c(u)$, where $c(u)$ is increasing on $(-\infty, 0)$ and decreasing on $(0, \infty)$. 
Example 3. The $\operatorname{NIG}(\alpha, \beta, \mu, \delta)$ law is self-decomposable, as follows from the representation (23) and the fact that the Bessel function $K_{1}$ is decreasing.

Let $y$ be a self-decomposable random variable with Lévy measure $Q$ of the form $Q(\mathrm{~d} u)=$ $q(u) \mathrm{d} u$ with $q(u)=|u|^{-1} c(u)$. As it is self-decomposable, $y$ is representable as

$$
y=\int_{0}^{\infty} \mathrm{e}^{-s} \mathrm{~d} \grave{z}(s)
$$

where $z$ is a Lévy process whose law is determined uniquely by that of $y$. The Lévy measure $W$ of $\grave{z}(1)$ is related to the Lévy density $q$ of $y$ by the formula

$$
W^{+}(u)=W([u, \infty))=u q(u)
$$

for $u>0$, and

$$
W^{-}(u)=W((-\infty, u])=|u| q(u)
$$

for $u<0$.

Furthermore, if the Lévy density $q$ of $y$ is differentiable, then $W$ has density $w$ - with respect to Lebesgue measure - given by

$$
w(u)=-q(u)-u q^{\prime}(u),
$$

where a prime denotes differentiation. The process $\grave{z}(u), u \geq 0$, is termed the background driving Lévy process (BDLP) corresponding to $y$.

In the following, $y$ will stand for a self-decomposable random variable whose Lévy density $q$ is differentiable, and $z(t), t \geq 0$, will denote the Lévy process generated by $y$ (see Section 3 ), i.e. the Lévy process such that $z(1) \stackrel{L}{=} y$. The BDLP determined by $z(1)$ is denoted by $z$. The BDLP of $z(u)$ is equivalent in law to $\grave{z}(u s), s \geq 0$.

We will use the notation

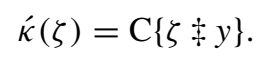

For any $u>0$ and $\lambda>0$, we may rewrite the representation (22) as

$$
y=\int_{0}^{\infty} \mathrm{e}^{-\lambda s} \mathrm{~d} \grave{z}(\lambda s)=\int_{0}^{u} \mathrm{e}^{-\lambda s} \mathrm{~d} \grave{z}(\lambda s)+\mathrm{e}^{-\lambda u} \int_{0}^{\infty} \mathrm{e}^{-\lambda s} \mathrm{~d} \grave{z}(\lambda(s+u))
$$

and, here,

$$
\int_{0}^{\infty} \mathrm{e}^{-\lambda s} \mathrm{~d} \grave{z}(\lambda(s+u)) \stackrel{L}{=} y .
$$

Consequently, $y$ is representable as

$$
y=\mathrm{e}^{-\lambda u} y_{0}+w_{u},
$$

where $y_{0}$ and $w_{u}$ are independent, $y_{0} \stackrel{L}{=} y$, and

$$
w_{u}=\int_{0}^{u} \mathrm{e}^{-\lambda s} \mathrm{~d} \grave{z}(\lambda s)
$$




\subsection{Ornstein-Uhlenbeck-type processes}

In fact, a stronger statement is true (see, e.g. Barndorff-Nielsen (1998b) and references therein): for any $\lambda>0$, the stochastic differential equation

$$
\mathrm{d} y(u)=-\lambda y(u) \mathrm{d} u+\mathrm{d} \grave{z}(\lambda u)
$$

has a stationary solution $y(u)$ such that $y(u) \stackrel{L}{=} y$. This stochastic differential equation is solved by

$$
y(u)=\mathrm{e}^{-\lambda u} y(0)+\int_{0}^{u} \mathrm{e}^{-\lambda(u-s)} \mathrm{d} \grave{z}(\lambda s) .
$$

A stationary process $y(u)$ of this kind is said to be an Ornstein-Uhlenbeck-type (OU-type) process. When $y(u)$ is square integrable with $\mathrm{E} y(u)=0$, it has correlation function $r(u)=$ $\exp \{-\lambda u\}, u>0$.

The stationary process $y(u), u \geq 0$, can be extended to a stationary process on the whole real line. To do so, we introduce an independent copy of the process $\grave{z}^{(\lambda)}(u) \stackrel{L}{=} z(\lambda u), u \geq 0$, but modify it to be càglàd, i.e. continuous from the left, with limits from the right, thus obtaining a process $\grave{z}_{1}^{(\lambda)}(u)$. Now, for $u<0$, respectively define $\grave{z}^{(\lambda)}(u)$ and $y(u)$ by $\grave{z}^{(\lambda)}(u)=\grave{z}_{1}^{(\lambda)}(-u)$ and

$$
y(u)=\mathrm{e}^{-\lambda|u|} y(0)+\mathrm{e}^{-\lambda|u|} \int_{u}^{0} \mathrm{e}^{\lambda|s|} \mathrm{d} \grave{z}^{(\lambda)}(s) .
$$

Then, $\grave{z}^{(\lambda)}(u), u \in \mathbb{R}$, is a (càdlàg) Lévy process and $y(u), u \in \mathbb{R}$, is a strictly stationary process of OU type with correlation function (if it exists)

$$
r(u)=\mathrm{e}^{-\lambda|u|}, \quad u \in \mathbb{R} .
$$

The integrated process can be represented as follows:

$$
S_{\varepsilon}(x)=\int_{0}^{x / \varepsilon} y(u) \mathrm{d} u=\frac{1}{\lambda}\left\{\grave{z}^{(1)}\left(\lambda \frac{x}{\varepsilon}\right)-y\left(\frac{x}{\varepsilon}\right)+y(0)\right\} .
$$

Example 4. The $\operatorname{NIG}(\alpha, \beta, \mu, \delta)$ distribution is self-decomposable. Thus, there exists a stationary OU-type process $y(u), u \in \mathbb{R}$, such that $y(u) \sim \operatorname{NIG}(\alpha, \beta, \mu, \delta)$ for every $u \in \mathbb{R}$, whatever the value of the parameter $\lambda>0$, and with correlation function (24). For $\mu=\beta=0$, we have $\mathrm{E} y(u)=0$.

First, consider the OU-type stationary process $y(u), u \in \mathbb{R}$, with marginal $\operatorname{NIG}(\alpha, 0,0, \delta)$ and correlation function $r_{y}(u)=\mathrm{e}^{-|u|}, u \in \mathbb{R}$. It is easy to see that $\varepsilon^{1 / 2} y(x / \varepsilon) \rightarrow 0$, $\varepsilon^{1 / 2} y(0) \rightarrow 0$ in probability as $\varepsilon \rightarrow 0$, and

$$
\begin{aligned}
\lim _{\varepsilon \rightarrow 0} \operatorname{Eexp}\left\{i \zeta \sqrt{\varepsilon} \grave{z}^{(1)}\left(\frac{x}{\varepsilon}\right)\right\} & =\lim _{\varepsilon \rightarrow 0}\left[\operatorname{Eexp}\left\{\mathrm{i} \zeta \sqrt{\varepsilon} \grave{z}^{(1)}(1)\right\}\right]^{x / \varepsilon} \\
& =\exp \left\{-\frac{1}{2} \zeta^{2} x \sigma^{2}\right\}, \\
\sigma^{2} & =\mathrm{E}\left[\grave{z}^{(1)}(1)\right]^{2}, \quad \zeta \in \mathbb{R} .
\end{aligned}
$$

Then by Slutsky's lemma, as $\varepsilon \rightarrow 0$,

$$
\sqrt{\varepsilon} \int_{0}^{x / \varepsilon} y(u) \mathrm{d} u \rightarrow \sigma b(x), \quad x \geq 0,
$$


in the sense of convergence of finite-dimensional distributions, where $b(x), x \geq 0$, is standard Brownian motion.

A modification of the above argument shows that, for the OU-type stationary process $y(u)$, $u \in \mathbb{R}$, with marginal $\operatorname{NIG}(\alpha, \beta, 0, \delta)$ distribution and correlation function $r_{y}(u)=\mathrm{e}^{-\lambda|u|}$, $u \in \mathbb{R}$, we have, as $\varepsilon \rightarrow 0$,

$$
\sqrt{\varepsilon} \int_{0}^{x / \varepsilon}\left[y(u)-\frac{\delta \beta}{\sqrt{\alpha^{2}-\beta^{2}}}\right] \mathrm{d} u \rightarrow \sigma ́ \sigma b(x), \quad x \geq 0,
$$

in the sense of convergence of finite-dimensional distributions, where

$$
\hat{\sigma}^{2}=\frac{\alpha^{2} \delta}{\lambda\left(\alpha^{2}-\beta^{2}\right)^{3 / 2}}
$$

\subsection{SupOU processes}

A SupOU process is a stochastic process obtained by superposition (i.e. addition) of independent OU-type processes. The stationary process with NIG marginal distribution and long-range dependence considered in Theorem 5, next, is a supOU process that was introduced in Barndorff-Nielsen (1998a).

Let $\vartheta(s)=\sum_{k=1}^{\infty} k^{-s}, s>1$, be the Riemann zeta function.

Theorem 5. Let $y^{(k)}(u), u \in \mathbb{R}, k=1,2, \ldots$, be a sequence of independent OU-type processes such that, for all $k$ and $u \in \mathbb{R}$, the marginal distribution of $y^{(k)}(u)$ is $\operatorname{NIG}\left(\alpha, \beta, 0, \delta_{k}\right)$ with

$$
\delta_{k}=\frac{1}{k^{1+2(1-H)}}, \quad k=1,2, \ldots, H \in(0,1),
$$

and let

$$
\delta=\sum_{k=1}^{\infty} \delta_{k}=\vartheta(1+2(1-H)) .
$$

Suppose, moreover, that the processes $y^{(k)}(u)$ all have the correlation function (24).

Then, the process

$$
y(u)=\sum_{k=1}^{\infty} y^{(k)}\left(k^{-1} u\right), \quad u \in \mathbb{R},
$$

is stationary and well defined as an $L_{2}$ limit, the marginal distribution of $y(u)$ is $\mathrm{NIG}(\alpha, \beta, 0, \delta)$ (where $\delta$ is defined as in (26)), the correlation function of the process $y(u)$ is of the form

$$
\begin{aligned}
\bar{r}(\tau) & =[\vartheta(1+2(1-H))]^{-1} \sum_{k=1}^{\infty} \frac{\mathrm{e}^{-|\tau| \lambda / k}}{k^{1+2(1-H)}} \\
& =\frac{1}{|\tau|^{2(1-H)}} \frac{\Gamma(2(1-H))}{\lambda^{2(1-H)} \vartheta(1+2(1-H))}(1+o(1)), \quad \frac{1}{2}<H<1,
\end{aligned}
$$

as $|\tau| \rightarrow \infty$, and the normalized spectral density is

$$
\bar{f}(\mu)=\frac{(\lambda / \pi)}{\vartheta(1+2(1-H))} \sum_{k=1}^{\infty} \frac{1}{k^{2(1-H)}} \frac{1}{\left(\lambda^{2}+\mu^{2} k^{2}\right)}, \quad \mu \in \mathbb{R} .
$$


Furthermore, the normalized integrated process

$$
\begin{aligned}
Y_{\varepsilon}(s) & =\varepsilon^{H} \int_{0}^{s / \varepsilon}\left[y(u)-\frac{\delta \beta}{\sqrt{\alpha^{2}-\beta^{2}}}\right] \mathrm{d} u \rightarrow \sigma_{0} b_{H}(s), \quad s \geq 0, \quad \text { where } \\
\sigma_{0}^{2} & =\frac{\delta \alpha^{2} \Gamma(2(1-H))}{\left(\alpha^{2}-\beta^{2}\right)^{3 / 2} \vartheta(1+2(1-H)) \lambda^{2(1-H)} H(2 H-1)} .
\end{aligned}
$$

Here, convergence is in the sense of finite-dimensional distributions as $\varepsilon \rightarrow 0$, and $b_{H}(s)$, $s \geq 0$, is fractional Brownian motion with fractional index $H$, i.e. a Gaussian process with zero mean and correlation function

$$
\frac{1}{2}\left\{|s|^{2 H}+|t|^{2 H}-|t-s|^{2 H}\right\}
$$

Sketch of proof. For simplicity, we put $\lambda=1$ and $\beta=0$. Then, from (26), we obtain

$$
\begin{aligned}
\operatorname{var} \int_{0}^{s / \varepsilon} y(u) \mathrm{d} u & =\frac{\operatorname{var} y(0)}{\vartheta(1+2(1-H))} \int_{0}^{s / \varepsilon} \int_{0}^{s / \varepsilon}\left[\sum_{k=1}^{\infty} \delta_{k} \mathrm{e}^{-|u-w| / k}\right] \mathrm{d} u \mathrm{~d} w \\
& =\frac{\operatorname{var} y(0)}{\vartheta(1+2(1-H)) H(2 H-1)} \Gamma(2(1-H))(s / \varepsilon)^{2 H}(1+o(1))
\end{aligned}
$$

because

$$
F(\tau)=\sum_{k=1}^{\infty} \delta_{k} \mathrm{e}^{-|\tau| / k}=\frac{F^{*}(\tau)}{|\tau|^{2(1-H)}}
$$

where, letting $\gamma=2(1-H) \in(0,1)$,

$$
F^{*}(\tau)=\tau^{2(1-H)} \sum_{k=1}^{\infty} \frac{1}{k^{1+2(1-H)}} \mathrm{e}^{-\tau / k} \sim \tau^{\gamma} \int_{1}^{\infty} x^{-1-\gamma} \mathrm{e}^{-\tau / x} \mathrm{~d} x=\int_{0}^{\tau} \mathrm{e}^{-y} y^{\gamma-1} \mathrm{~d} y
$$

as $\tau \rightarrow \infty$.

Statement (30) can now be proved by such standard methods as the discretization procedure and the Lindeberg-Feller central limit theorem.

Remark 3. The spectral density (29) satisfies the following asymptotic relations:

$$
\bar{f}(\mu)=\frac{c_{1}}{|\mu|^{1-2(1-H)}}(1+o(1)), \quad|\mu| \rightarrow 0, \frac{1}{2}<H<1,
$$

and

$$
\bar{f}(\mu)=\frac{c_{2}}{|\mu|^{2}}(1+o(1)), \quad|\mu| \rightarrow \infty,
$$

where $c_{1}, c_{2}>0$ are some constants. Equation (32) indicates long-range dependence.

\section{Scaling laws: NIG-OU and NIG-supOU initial conditions}

As was shown in Section 3, the limiting behavior of the average processes (16), (18), and (20) is the same, respectively, as that of the average processes (17), (19), and (21). 
From (25) and Theorems 2-5, we obtain Theorem 6, as follows.

Theorem 6. Let $u(t, x), t>0, x \in \mathbb{R}$, be a solution of the initial-value problem (1), (4) in which $\xi(x), x \in \mathbb{R}$, is a stationary random process that has locally integrable sample paths. Suppose that one of the following conditions holds:

(a) $\xi(x), x \in \mathbb{R}$, is an OU-type process with marginal $\operatorname{NIG}(\alpha, \beta, 0, \delta)$ distribution and correlation function (24);

(b) $\xi(x), x \in \mathbb{R}$, is a supOU process with zero mean, correlation function (28), and marginal $\mathrm{NIG}(\alpha, \beta, 0, \delta)$ distribution as described in Theorem 5.

Then, for $\varepsilon \rightarrow 0$, we have the following convergence of random processes, in the sense of finite-dimensional distributions.

(i) If the external potential vanishes, i.e. $\Phi=0$, then

$$
\varepsilon^{1 / 2} \int_{0}^{x / \varepsilon}\left[u(t, y)-\frac{\delta \beta}{\sqrt{\alpha^{2}-\beta^{2}}}\right] \mathrm{d} y \rightarrow \dot{\sigma} b(x), \quad x \geq 0,
$$

when (a) holds, where $\sigma^{2}$ is as defined in (25) and $b$ is Brownian motion. When (b) holds,

$$
\varepsilon^{H} \int_{0}^{x / \varepsilon}\left[u(t, y)-\frac{\delta \beta}{\sqrt{\alpha^{2}-\beta^{2}}}\right] \mathrm{d} y \rightarrow \sigma_{0} b_{H}(x), \quad x \geq 0, H \in\left(\frac{1}{2}, 1\right),
$$

where $\sigma_{0}^{2}$ is as defined in (31) and $b_{H}$ is fractional Brownian motion.

(ii) If the external potential $\Phi$ is linear, as in (9), then, when (a) holds,

$$
\varepsilon^{1 / 2} \int_{0}^{x / \varepsilon}\left[u(t, y)-2 t b \mu-\frac{\delta \beta}{\sqrt{\alpha^{2}-\beta^{2}}}\right] \mathrm{d} y \rightarrow \dot{\sigma} b(x), \quad x \geq 0,
$$

while, when (b) holds,

$$
\varepsilon^{H} \int_{0}^{x / \varepsilon}\left[u(t, y)-2 t b \mu-\frac{\delta \beta}{\sqrt{\alpha^{2}-\beta^{2}}}\right] \mathrm{d} y \rightarrow \sigma_{0} b_{H}(x), \quad x \geq 0, H \in\left(\frac{1}{2}, 1\right) .
$$

(iii) If the external potential $\Phi$ is quadratic, as in (11), then, when (a) holds,

$$
\begin{aligned}
& \varepsilon^{1 / 2} \int_{0}^{x / \varepsilon}\left[u(t, y)-2 y \sqrt{b \mu} \tanh (\omega t)-\frac{\delta \beta}{\cosh (\omega t) \sqrt{\alpha^{2}-\beta^{2}}}\right] \mathrm{d} y \\
& \rightarrow \sigma ́ \sigma\left(\frac{x}{\cosh (\omega t)}\right), \quad x \geq 0,
\end{aligned}
$$

while, when (b) holds,

$$
\begin{aligned}
\varepsilon^{H} \int_{0}^{x / \varepsilon}[u(t, y)-2 y \sqrt{b \mu} \tanh (\omega t) & \left.-\frac{\delta \beta}{\cosh (\omega t) \sqrt{\alpha^{2}-\beta^{2}}}\right] \mathrm{d} y \\
& \rightarrow \sigma_{0} b_{H}\left(\frac{x}{\cosh (\omega t)}\right), \quad x \geq 0, H \in\left(\frac{1}{2}, 1\right) .
\end{aligned}
$$

From Theorem 6, we can see the differences and similarities between the homogeneous and inhomogeneous Burgers turbulence problems when the external potential is linear or quadratic. More specifically, the inhomogeneous problem contains some trends or centering functions under the averaging and, in the quadratic-potential case, the limiting processes depend on time. 


\section{Acknowledgements}

The first author gratefully acknowledges financial support for this work from the Danish National Research Foundation and the EPSRC grant RCMT091. The second author was partially supported by the Australian Research Council grant DP 0345577 and the EPSRC grant RCMT091. We would also like to thank the referee, who kindly pointed out to us some important missing references.

\section{References}

Albeverio, S., Molchanov, S. A. and Surgailis, D. (1994). Stratified structure of the universe and Burgers' equation-a probabilistic approach. Prob. Theory Relat. Fields 100, 457-484.

ANH, V. V. AND LeONENKo, N. N. (1999). Non-Gaussian scenarios for the heat equation with singular initial conditions. Stoch. Process Appl. 84, 91-114.

Barndorff-Nielsen, O. E. (1998a). Probability and statistics, self-decomposability, finance and turbulence. In Probability Towards 2000 (Lecture Notes Statist. 128), eds L. Accardi and C. C. Heyde, Springer, New York, pp. 47-57.

BARNDORFF-NiElsen, O. E. (1998b). Processes of normal inverse Gaussian type. Finance Stoch. 2, 41-68.

Barndorff-Nielsen, O. E. (2001). Superposition of Ornstein-Uhlenbeck type processes. Theory Prob. Appl. 45, 175-194.

Barndorff-Nielsen, O. E. ANd Pérez-Abreu, V. (1999). Stationary and self-similar processes driven by Lévy processes. Stoch. Process Appl. 84, 357-369.

Batchelor, G. (1953). The Theory of Homogeneous Turbulence. Cambridge University Press.

Berline, N., Getzler, E. And Vergne, M. (1992). Heat Kernels and Dirac Operators. Springer, Berlin.

Bertini, L. AND CANCRINI, N. (1995). The stochastic heat equation: Feynman-Kac formula and intermittence. J. Statist. Phys. 78, 1377-1401.

Bertoin, J. (1998a). Large-deviation estimates in Burgers turbulence with stable noise initial data. J. Statist. Phys. 91, 655-667.

Bertoin, J. (1998b). The inviscid Burgers equation with Brownian initial velocity. Commun. Math. Phys. 193, $397-406$.

BulinskiĬ, A. V. AND Molchanov, S. A. (1991). Asymptotic Gaussian property of the solution of the Burgers equation with random initial data. Theory Prob. Appl. 36, 217-236.

Burgers, J. (1974). The Nonlinear Diffusion Equation. Kluwer, Dordrecht.

Chorin, A. J. (1975). Lectures on Turbulence Theory. Publish or Perish, Berkeley, CA.

Cycon, H. L., Froese, R. G., Kirsch, W. And Simon, B. (1987). Schrödinger Operators. Springer, Berlin.

Deriev, I. And Leonenko, N. (1997). Limit Gaussian behavior of the solutions of the multidimensional Burgers' equation with weak-dependent initial conditions. Acta Appl. Math. 47, 1-18.

Dermone, A., Hamadene, S. And Ouknine, Y. (1999). Limit theorems for statistical solution of Burgers equation. Stoch. Process. Appl. 81, 17-230.

Dobrushin, R. L. AND Major, P. (1979). Major non-central limit theorems for nonlinear functionals of Gaussian fields. Z. Wahrscheinlichkeitsth. 50, 27-52.

Frisch, U. (1995). Turbulence. Cambridge University Press.

Funaki, T., Surgailis, D. AND WoyCZYŃSKI, W. A. (1995). Gibbs-Cox random fields and Burgers turbulence. Ann. Appl. Prob. 5, 461-492.

Gurbatov, S., Malakhov, A. And Saichev, A. (1991) Non-linear Waves and Turbulence in Nondispersive Media: Waves, Rays and Particles. Manchester University Press.

Hodges, S. AND CARVerhill, A. (1993). Quasi mean reversion in an efficient stock market: the characterization of economic equilibria which support Black-Scholes option pricing. Econom. J. 103, 395-405.

Hodges, S. And Selby, M. J. P. (1997). The risk premium in trading equilibria which support Black-Scholes option pricing. In Mathematics of Derivative Securities, eds M. Dempster and S. Pliska, Cambridge University Press, pp. 41-53.

Holden, M., Øкsendal, B., Ubøe, J. And Zhang, T. S. (1996). Stochastic Partial Differential Equations: A Modelling, White Noise Functional Approach. Birkhäuser, Boston, MA.

Hopf, E. (1950). The partial differential equation $u_{x}+u u_{x}=\mu u_{x x}$. Commun. Pure Appl. Math. 3, 201-230.

Hu, Y. AND WoYCZYŃski, W. A. (1995). Limiting behaviour of quadratic forms of moving averages and statistical solutions of the Burgers' equation. J. Multivariate Anal. 52, 15-44.

Kardar, M., Parisi, G. And Zhang, Y. C. (1986). Dynamical scaling of growing interfaces. Phys. Rev. Lett. 56, 889-892.

KochmansKi, S. (1994). On the evolution operators for some equations of mathematical physics with variable coefficients. Ukranian Math. J. 46, 938-952. 
LeOnenko, N. (1999). Limit Theorems for Random Fields with Singular Spectrum. Kluwer, Dordrecht.

LEONENKo, N. N. AND LI, Z. B. (1994). Non-Gaussian limit distributions of solutions of Burgers equation with strongly dependent random initial conditions. Random Oper. Stoch. Equations 2, 95-102.

LeOnenko, N. N. ANd Mel'Nikova, O. O. (2001). Rescaling and homogenization of solutions of the heat equation with a linear potential and of the corresponding Burgers equation with random data. Theory Prob. Math. Statist. 62, 77-88.

LeOnenko, N. AND ORSINGHeR, E. (1995). Limit theorems for solutions of Burgers equation with Gaussian and non-Gaussian initial data. Theory Prob. Appl. 40, 387-403.

LEONEnKo, N. N. AND WoyCZYŃsKi, W. A. (1998). Exact parabolic asymptotics for singular $n-D$ Burgers' random fields: Gaussian approximation. Stoch. Process. Appl. 76, 141-165.

Molchanov, S. A., Surgailis, D. And WoyczyńsKi, W. A. (1995). Hyperbolic asymptotics in Burgers turbulence. Commun. Math. Phys. 168, 209-226.

Molchanov, S. A., Surgailis, D. And Woyczyński, W. A. (1997). The large-scale structure of the universe and quasi-Voronoi tessellation of shock fronts in forced Burgers turbulence in $\boldsymbol{R}^{d}$. Ann. Appl. Prob. 7, 220-223.

Rosenblatt, M. (1987). Scale renormalization and random solutions of Burgers equation. J. Appl. Prob. 24, 328-338.

RYAN, R. (1998). The statistics of Burgers turbulence initiated with fractional Brownian noise data. Commun. Math. Phys. 191, 1008-1038.

SAichev, A. I. AND Woyczyński, W. A. (1997). Evolution of Burgers' turbulence in the presence of external forces. J. Fluid Mech. 331, 313-343.

Shandarin, S. F. ANd Zeldovich, YA. B. (1989). Turbulence, intermittency, structures in a left-gravitating medium: the large scale structure of the universe. Rev. Modern Phys. 61, 185-220.

SINAI, YA. G. (1992). Statistics of shocks in solutions of inviscid Burgers equation. Commun. Math. Phys. 148, 601-621.

TAQQU, M. S. (1975). Weak convergence to fractional Brownian motion and to the Rosenblatt process. Z. Wahrscheinlichkeitsth. 31, 287-302.

TAQQU, M. S. (1979). Convergence of integrated processes of arbitrary Hermite rank. Z. Wahrscheinlichkeitsth. 50, 53-83.

Watson, G. N. (1944). A Treatise of the Theory of Bessel Functions. Cambridge University Press.

Witham, G. B. (1974). Linear and Nonlinear Waves. John Wiley, New York.

WoyCZYŃski, W. A. (1998). Burgers-KPZ Turbulence (Lecture Notes Math. 1706). Springer, Berlin. 